\title{
De la traducción a la enseñanza de lenguas extranjeras como actividades mediadas culturalmente
}

\author{
Ana Isabel CAPEL MORENO \\ anai.capel@gmail.com \\ Círculo Andaluz de Lingüística aplicada \\ de la Universidad de Almería
}

Resumen: El presente artículo pretende establecer una relación entre la traducción profesional y la enseñanza de lenguas extranjeras entendidas como actividades en las que interviene la mediación cultural. En primer lugar, se analiza un trabajo del profesor Emilio Ortega en el que expone las razones para afirmar que la traducción es una actividad mediada culturalmente. Posteriormente se consideran los aspectos en los que pueden confluir la mediación cultural en traducción y en enseñanza de lenguas. Finalmente, se analiza un estudio sobre la mediación cultural en las aulas australianas. Como conclusión se destaca que al igual que en traducción, el conocimiento cultural es esencial en la actividad de los profesores de lenguas y se señalan futuras líneas de investigación en este campo.

Palabras clave: mediación cultural, enseñanza de lenguas extranjeras, traducción 


\section{A. I. Capel Moreno}

2019. "De la traducción a la enseñanza de lenguas extranjeras como actividades mediadas culturalmente" Sabir. International Bulletin of Applied Linguistics, 1: 38-51

Abstract: This article attempts to research the relationship between professional translation and foreign language teaching considering that both are activities in which cultural mediation occurs. The first part refers to the considerations that lead Professor Emilio Ortega to state that translation is a culturally mediated activity. Afrterwards, the common areas in which cultural mediation could take place in translation and in language teaching are analised. Finally, a study about cultural mediation in Australian classrooms is reviewed. The article concludes with the idea that cultural knowledge is essential both in translation and in language teaching and the indication of future areas of research in this field.

Keywords: cultural mediation, foreign language teaching, translation

Este trabajo pretende analizar las conclusiones del profesor Emilio Ortega (2004) en su artículo: "La traducción como actividad mediada culturalmente: revisión crítica de la Tesis de la indeterminación de la traducción de Quine" y relacionar dichas conclusiones con el ámbito de la enseñanza de lenguas extranjeras en el panorama actual. Si bien el artículo citado se circunscribe al ámbito de la traducción especializada, la relación entre esta disciplina y la didáctica de lenguas es evidente y ha sido abordada desde distintos enfoques desde mediados del siglo XX hasta la actualidad. De hecho, E. Ortega (2004:6164) antes de adentrarse en la importancia del factor cultural en el proceso de traducción establece la consideración preliminar de que la Traductología es una disciplina de anclaje mustidisciplinar y expone cómo la necesidad de comunicación entre distintos Estados tras la II Guerra Mundial "supone una eclosión de los estudios traductológicos y lingüísticos (...) y, sobre todo, para el nacimiento de la Lingüística Aplicada (...) y el desarrollo imparable de la Metodología de enseñanza/aprendizaje de Lenguas Extranjeras". Sin duda alguna 


\section{A. I. Capel Moreno}

2019. "De la traducción a la enseñanza de lenguas extranjeras como actividades mediadas culturalmente" Sabir. International Bulletin of Applied Linguistics, 1: 38-51

dicha necesidad de comunicación entre hablantes de distintas lenguas sigue vigente hoy en día y constituye un punto de intersección entre la traducción y la enseñanza-aprendizaje de lenguas extranjeras (LEs). Sin embargo, la comunicación entre hablantes de dos lenguas no es posible simplemente haciendo uso de los sistemas morfológicos, sintácticos y fonéticos que las regulan. El conocimiento de la realidad sociocultural que acompaña a ambas lenguas es fundamental para que dicha comunicación sea efectiva siendo este conocimiento un interés común en el ámbito de la Traductología y de la Lingüística Aplicada a la enseñanza de lenguas.

Este artículo tomará como punto de partida el mencionado artículo de E. Ortega en el que argumenta por qué la traducción es una actividad mediada culturalmente con el fin de establecer las analogías oportunas en el campo de enseñanza de LEs. A continuación, se considera en qué puntos puede confluir la mediación cultural en traducción y en enseñanza de LEs según el marco intercultural de enseñanza de lenguas que propone Butjjer (1991). Finalmente, se hace un análisis de un estudio empírico sobre mediación cultural en el aula de LEs llevado a cabo por Michelle Kohler en Australia. El objetivo es no sólo identificar la confluencia en mediación cultural entre traducción y enseñanza de lenguas en el plano teórico, sino también conocer los resultados que arroja un estudio reciente en este campo, además de identificar futuras líneas de investigación en mediación cultural en la didáctica de LEs.

\section{La mediación cultural en Traducción. Vinculación con la enseñanza de Les}

El artículo que nos ocupa de E. Ortega, que comienza con una revisión del fenómeno traductor y de la evolución de la Traductología como disciplina, refuta la tesis de Quine (1959) sobre "la indeterminación de la traducción" desde una 


\section{A. I. Capel Moreno}

2019. "De la traducción a la enseñanza de lenguas extranjeras como actividades mediadas culturalmente" Sabir. International Bulletin of Applied Linguistics, 1: 38-51

perspectiva contemporánea. Quine exponía una situación de traducción radical entre un lingüista (entendido como traductor) y un aborigen "en la que los interlocutores no tienen puntos de referencia en los que basar su intercambio comunicativo" dándose por tanto la situación de imposibilidad de traducción. E. Ortega (2004:67) afirma que dicha tesis resulta inviable desde un punto de vista traductológico teniendo en cuenta (entre otros factores) que "nunca se plantea que un traductor vaya a enfrentarse a una situación de traducción radical porque la traducción sólo es posible cuando se tiene conocimiento de ambas comunidades lingüísticas y de las convenciones culturales y sociales que las conforman." Esta afirmación es totalmente aplicable a la enseñanza de lenguas dado que un enseñante que ignorase el componente cultural en el aula sería un mero transcodificador lingüístico, tal y como se entendía en el método didáctico gramática-traducción. Además de esta premisa fundamental, que sintetiza la argumentación hacia la tesis de Quine, extraemos tres conclusiones del artículo de Ortega (2004:71-72) que pueden ser aplicables al ámbito de enseñanza de LEs. Por una parte, el hecho de que resulta imprescindible considerar el uso de la lengua "desde una perspectiva de comunicación sustentada en la existencia de convenciones socioculturales que condicionan y determinan la emisión de proferencias lingüísticas en función de los contextos situacionales que se presenten y de la finalidad que guíe ese establecimiento de un acto de habla". Por otra parte, que "en cuanto a la adecuación/inadecuación de una proferencia lingüística existe un entramado de convenciones socioculturales" que determinarán la pertinencia de uso en función del sistema de creencias compartidas por una determinada sociedad. Por último, "la necesidad de abordar la problemática lingüística desde una perspectiva compleja e interdisciplinar en la que no sólo intervienen mecanismos de estímulo-respuesta, sino que se trata de un proceso complejo (psicológico, sociológico y cultural) (...)". En resumen, para E. Ortega: 


\section{A. I. Capel Moreno}

2019. "De la traducción a la enseñanza de lenguas extranjeras como actividades mediadas culturalmente" Sabir. International Bulletin of Applied Linguistics, 1: 38-51

1. No sólo es necesario el conocimiento lingüístico de las dos lenguas entre las que se traduce, sino que también hay que conocer las convenciones culturales y sociales de sus hablantes.

2. El conocimiento sociocultural determina que se tengan que emplear distintas expresiones o fórmulas según las situaciones dadas y el fin que se desea conseguir para que resulten adecuadas en la Lengua Meta. Por lo tanto, este conocimiento es esencial para que tenga lugar la adecuación lingüística ${ }^{1}$ entendida como:

"la propiedad textual por la que el texto se adapta al contexto discursivo. Quiere esto decir que el texto se amolda a los interlocutores, a sus intenciones comunicativas, al canal de producción y recepción, etc., parámetros todos ellos que definen los registros. Por tanto, un texto es adecuado si la elección lingüística efectuada es apropiada a la situación comunicativa."

3. Los procesos psicológicos, sociológicos y culturales deberán ser tenidos en cuenta también al considerar los fenómenos lingüísticos desde una perspectiva interdisciplinar.

Las consideraciones que se están haciendo en torno a la actividad del traductor se basan en el conocimiento lingüístico y sociocultural de dos lenguas. Dichas consideraciones serán también aplicables al aula de LEs siempre y cuando el alumnado comparta la misma lengua materna (LM) y por lo tanto el mismo sistema de convenciones socioculturales anteriormente mencionado tal y como

\footnotetext{
1 Centro Virtual Cervantes: Diccionario de términos clave de ELE. "Adecuación" https://cvc.cervantes.es/ensenanza/biblioteca_ele/diccio_ele/diccionario/adecuacion.ht m [consulta 9 octubre 2019].
} 


\section{A. I. Capel Moreno}

2019. "De la traducción a la enseñanza de lenguas extranjeras como actividades mediadas culturalmente" Sabir. International Bulletin of Applied Linguistics, 1: 38-51

es el caso de muchos centros bilingües en el territorio europeo. Si se da esta circunstancia, el profesor puede tender un puente en el aula entre los sistemas de convenciones socioculturales que acompañan a la $L M$ y la $L E$, guiar al alumnado en la toma de decisiones sobre la adecuación/inadecuacción de las proferencias lingüísticas emitidas desde o hacia la LE en los contextos dados y desde un punto de vista pragmalingüístico, arrojar luz sobre los factores sicológicos, sociológicos y culturales que intervienen en la comunicación no sólo en la LE sino también en la propia LM. Desde el punto de vista de la mediación cultural, no obstante, la diferencia entre el papel del profesor y el traductor radica en que el primero utiliza las situaciones comunicativas en el aula para hacer explícitos los contrastes que existen entre las dos lenguas y sus convenciones socioculturales como instrumento de enseñanza/aprendizaje, mientras que la mediación cultural en la actividad del traductor es un proceso que no es explícito ni colectivo, sino implícito e individual (en la mayoría de las ocasiones), y quedará reflejado en el texto meta o resultado final de la traducción.

\section{La teoría intercultural de enseñanza de lenguas. Conexiones con la traducción}

El interés por el componente cultural desde la didáctica de lenguas, según Buttjes (1991:11), comenzó en Estados Unidos en la década de los setenta. Este interés también llegó a Europa en la década de los ochenta donde comenzaron las investigaciones sobre la mediación del lenguaje y la cultura en la clase de LEs en Gran Bretaña (Loveday:1982; Byram:1989), Francia (Zarate:1986) y Alemania Oriental (Wierlacher:1987) donde los estudios culturales e interculturales comenzaron por implantarse como disciplinas académicas. La importancia de la mediación no sólo lingüística, sino también cultural empezó a adquirir relevancia 


\section{A. I. Capel Moreno}

2019. "De la traducción a la enseñanza de lenguas extranjeras como actividades mediadas culturalmente" Sabir. International Bulletin of Applied Linguistics, 1: 38-51

en la enseñanza de LEs en el nuevo contexto de relaciones internacionales tras la creación de la Comunidad Europea. De hecho, el volumen "Mediating Languages and Cultures. Towards an Intercultural Theory of Foreign Language Education" (Buttjes y Byram:1991) recoge las contribuciones del simposium celebrado en Durham (1986) con el objetivo de tratar la cuestión cultural en la enseñanza de LEs. En la primera parte de dicho volumen, Buttjes (1991:1-16) propone un marco para el desarrollo de una teoría intercultural de la enseñanza de lenguas en el que encontramos las siguientes afirmaciones sobre la mediación cultural en LEs que coinciden con las conclusiones sobre mediación cultural en traducción según E. Ortega:

1. Se considera cada vez más frecuente que las dificultades en el aprendizaje de lenguas y el fracaso en la comunicación intercultural son de naturaleza cultural, más que lingüística (Buttjes 1991:7), por lo que al igual que en traducción, no sólo es necesario el conocimiento lingüístico de las dos lenguas empleadas, sino que también es necesario conocer las convenciones culturales y sociales de sus hablantes.

2. En el aula de LEs la competencia intercultural como tal puede referirse tanto a la información (cultural) como a las destrezas de mediación permitiendo la visión desde dentro de la cultura extranjera y fomentando la negociación del significado entre culturas (Buttjes 1991:9). El conocimiento sociocultural, por lo tanto, determina que se tengan que emplear distintas expresiones o fórmulas según las situaciones dadas y el fin que se desea conseguir para que resulten adecuadas en la lengua meta, lo que nos permite establecer una vinculación con el concepto de "adecuación" planteado por E. Ortega.

3. El enfoque interdisciplinar de los estudios socioculturales supone: que la información etnográfica es útil al diseñar materiales y formar profesores; que 


\section{A. I. Capel Moreno}

2019. "De la traducción a la enseñanza de lenguas extranjeras como actividades mediadas culturalmente" Sabir. International Bulletin of Applied Linguistics, 1: 38-51

la investigación y el aprendizaje deben incluir una evaluación crítica de los valores culturales y las disciplinas académicas; y que se deben considerar las opiniones expertas y las aportaciones internacionales al describir la realidad educativa o académica en un contexto sociohistórico (Buttjes 1991:12-13). Por lo tanto, se puede observar cómo los procesos psicológicos, sociológicos y culturales deberán ser tenidos en cuenta también al considerar los fenómenos lingüísticos desde una perspectiva interdisciplinar, tal y como se aludía en el ámbito de la traducción.

Las razones expuestas por las que se considera que tanto la traducción como la enseñanza de lenguas son dos actividades culturalmente mediadas permiten afirmar, volviendo a emplear las palabras de Ortega, que la Enseñanza de LEs, al igual que la traducción, "sólo es posible cuando se tiene conocimiento de ambas comunidades lingüísticas y de las convenciones culturales y sociales que las conforman". La diferencia radica en que el traductor normalmente hará uso de su conocimiento intercultural de manera individual vertiéndolo en el texto meta, mientras que el profesor compartirá dicho conocimiento en el aula, ya sea presentando información intercultural o guiando la elección de las fórmulas lingüísticas que resulten adecuadas en los contextos dados desde un punto de vista pragmático. Precisamente Zarate (1986) alude a esta necesidad de intervención del profesor debido a la diferencia que existe entre adquirir una cultura como extranjero y adquirir una cultura como hablante nativo siendo necesario en el primer caso que haya información explícita con respecto a los aspectos culturales.

Otra diferencia destacable entre traducción y didáctica de LEs es que la mediación cultural que tiene lugar en la actividad del traductor está determinada tanto por las características como por el contenido del texto que ha de traducir. El traductor no podrá eludir referentes culturales explícitos que aparezcan en el 


\section{A. I. Capel Moreno}

2019. "De la traducción a la enseñanza de lenguas extranjeras como actividades mediadas culturalmente" Sabir. International Bulletin of Applied Linguistics, 1: 38-51

texto original, y además deberá transmitir los matices relacionados con aspectos socioculturales que se presenten (tales como fórmulas de cortesía, slang, etc.). Sin embargo, el profesor de LEs sí tiene la posibilidad de controlar en qué medida se hace uso del conocimiento intercultural en el aula. El grado de control de la mediación cultural en una sesión de clase puede variar desde la mera búsqueda de un equivalente para una referencia cultural encontrada en un texto (por ejemplo, la búsqueda de un refrán con significado equivalente en otra lengua) hasta el debate en el aula sobre cuestiones políticas, sociales o temas de actualidad de los países donde se habla la LE, pasando por identificar los matices asociados al concepto de "variación lingüística", entendida como las "diferencias de uso de la lengua condicionado por factores de tipo geográfico, sociocultural, contextual o histórico"2 . La cantidad de exposición a la cultura extranjera, además, debería hacerse explicita en las programaciones de los programas de enseñanza y formar una parte relevante de los mismos con el fin de aprovechar que el factor cultural puede ser uno de los elementos más motivadores en el aprendizaje de una $\mathrm{LE}^{3}$.

\section{El profesor como mediador cultural en el aula de LE según M. Kohler}

Anteriormente nos hemos referido al contexto de enseñanza de LEs de la década de los ochenta cuando predominaba el enfoque comunicativo basado en un ideal monolingüe, en el que a menudo no se tenían en cuenta situaciones reales y propósitos de uso, lo que llevó a tomar conciencia de la importancia de

\footnotetext{
${ }^{2}$ Centro Virtual Cervantes. Diccionario de términos clave de ELE. "Variación Lingüística: Https://cvc.cervantes.es/ensenanza/biblioteca_ele/diccio_ele/diccionario/variacionlingui stica.htm [consulta 9 octubre 2019].

${ }^{3}$ Con respecto al tratamiento curricular de la competencia intercultural en España y en la Unión Europea véase Paricio Tato: 2014.
} 


\section{A. I. Capel Moreno}

2019. "De la traducción a la enseñanza de lenguas extranjeras como actividades mediadas culturalmente" Sabir. International Bulletin of Applied Linguistics, 1: 38-51

la relación entre la lengua y la cultura. Surge así el interés por la mediación cultural en el aula de LEs, que en palabras de Michelle Kohler (2015) se puede entender como "el acto de relacionar dos marcos lingüísticos y culturales con una finalidad educativa", y que ha sido estudiada por autores que reconocen la relación de la cultura y el contexto en el uso del lenguaje (Byram:1991; Nostrand:1991; Buttjes:1991) quienes han proporcionado marcos conceptuales y consideraciones prácticas que esta autora revisa en su libro "Teachers as Mediators in the FL Classroom" (Kohler:2015). Kohler revisa el concepto de "mediación" en el contexto de enseñanza de LEs, y entiende que un mediador actúa como "intermediario" usando su competencia para transferir significados de una parte a otra cuando esas partes no comparten la misma lengua. Según Kohler, la mediación desde este punto de vista es un proceso de aprender a "leer" un mero sistema lingüístico y cultural y transferir estos significados a otro sistema lingüístico y cultural. De esta forma la mediación se asocia con los procesos de traducción, paráfrasis y resumen. Desde el enfoque del aprendizaje sociocultural, sin embargo, la mediación ocurre cuando el profesor presenta al alumno nuevas informaciones e ideas que posibilitan la transformación de lo "nuevo" en lo "conocido". Kohler argumenta que en un contexto educativo formal el profesor es el principal mediador del aprendizaje y que en el aula de LEs el lenguaje representa el medio para el aprendizaje, así como también el objeto de aprendizaje. En dicho contexto, la autora estudia cómo los profesores pueden mediar desde una orientación intercultural. La mediación, por lo tanto, según esta autora es una dimensión integral de la enseñanza del lenguaje, un conjunto de prácticas y formas de ser que construye conexiones entre marcos existentes de los aprendices y nuevos marcos del lenguaje y culturales y al hacer esto, desarrollan también su propia capacidad de actuar como mediadores culturales. Uno de los marcos propuestos para abordar la mediación cultural, que habíamos empleado para establecer una comparación con la mediación en traducción, es 


\section{A. I. Capel Moreno}

2019. "De la traducción a la enseñanza de lenguas extranjeras como actividades mediadas culturalmente" Sabir. International Bulletin of Applied Linguistics, 1: 38-51

el de Buttjes (1991). Kohler: (op. cit.) hace referencia al intento que en dicho estudio se hace por realizar aportaciones teóricas y prácticas sobre mediación cultural, además de otros estudios que han estudiado distintas dimensiones del enfoque intercultural principalmente en lo relativo a currículos educativos, programación, recursos y en menor medida, evaluación. No obstante, existen pocas investigaciones (ver también Zarate et al. :2004) sobre la práctica en el aula y el papel del profesor como mediador en el aprendizaje intercultural de la lengua, por lo que tanto los resultados como los métodos de investigación que describe Kohler en su libro son especialmente interesantes. La investigación se llevó a cabo durante un año en Australia mediante estudios de caso con tres profesores de indonesio como LE con el fin de observar su mediación en el aprendizaje intercultural de la lengua de sus alumnos. Además de la dimensión individual de la experiencia de cada profesor, el estudio estaba dotado de una dimensión colectiva con el método "investigación-acción participativa", por el que los profesores intervenían en un ciclo basado en la planificación, la enseñanza y la reflexión sobre sus clases de LE. Se empleó la unidad didáctica como el instrumento para considerar una variedad de prácticas de mediación en una secuencia de enseñanza y aprendizaje. Los cuestionarios y documentos de trabajo empleados aparecen en el apéndice de la obra, lo que plantea la posibilidad de reproducir el estudio de la mediación cultural en otros contextos de enseñanza de LEs. En cuanto a la forma de proceder en el aula se empleó el análisis textual en textos auténticos para considerar la relación entre lengua y cultura. Con respecto a los datos de interacción en el aula, las formas de mediar se analizaron teniendo como referencia la mediación desde el punto de vista de la teoría del aprendizaje sociocultural y la enseñanza del lenguaje intercultural y se cruzaron los dos conjuntos de datos obtenidos: los conceptos que los profesores tenían sobre la relación entre lengua y cultura por un lado, y sus prácticas de mediación por otro lado, observándose una divergencia entre las consideraciones teóricas 


\section{A. I. Capel Moreno}

2019. "De la traducción a la enseñanza de lenguas extranjeras como actividades mediadas culturalmente" Sabir. International Bulletin of Applied Linguistics, 1: 38-51

de los profesores y su práctica docente. Como conclusión a su estudio, Kohler señala la importancia de permitir a los profesores desarrollar una capacidad reflexiva, de entender su papel como mediadores interculturales y reconocerse fundamentales para la mediación, además de apuntar futuras vías de investigación como la relación entre las formas de mediación de los profesores y la naturaleza del aprendizaje lingüístico y cultural que los alumnos desarrollan.

\section{Conclusiones}

El objetivo de este estudio ha sido establecer una conexión entre la traducción y la enseñanza de lenguas como actividades mediadas culturalmente tomando como punto de partida las reflexiones del artículo citado del profesor Emilio Ortega. Hemos llegado a la conclusión de que el conocimiento no sólo lingüístico, sino cultural es esencial en la actividad del traductor y del profesor de LEs. Los traductores acometen su tarea realizando un proceso de mediación cultural cuyo resultado se vierte en el texto meta. El profesor de LE, sin embargo, realiza dicha mediación en el aula. Las formas de hacerlo pueden ser más o menos explícitas, más o menos dirigidas, siendo los receptores de la mediación los aprendices de la LE. Según las investigaciones consultadas sobre el papel del profesor como guía del proceso de enseñanza-aprendizaje de una lengua extranjera es fundamental considerar la importancia del componente cultural que se asocia a dicha lengua con el fin de 1. Ampliar el conocimiento intercultural del alumnado intentando exponerles a las distintas variedades asociadas a un idioma tanto lingüísticas como socioculturales; 2. Facilitar el uso de repertorios lingüísticos que serán adecuados en las distintas situaciones comunicativas en cuyo caso, el uso contrastivo entre la LM y la LE puede ser muy útil para identificar los matices asociados a las fórmulas empleadas; 3 . Hacer explícitos los matices 


\section{A. I. Capel Moreno}

2019. "De la traducción a la enseñanza de lenguas extranjeras como actividades mediadas culturalmente" Sabir. International Bulletin of Applied Linguistics, 1: 38-51

sicológicos y sociales que comportan las proferencias lingüísticas, tanto en la LM como en la LE; 4. Realizar en el aula de LEs y fuera de ella actividades interculturales para exponer al alumnado a la realidad sociocultural de la LE que los profesores consideran tan importantes para el aprendizaje de una lengua.

Las líneas de investigación que se abren sobre la mediación cultural que los profesores llevan a cabo en el aula de LEs son amplias y entre otras, podrían dirigirse a replicar estudios como el de M. Kohler (2015) en distintos contextos educativos, a analizar del efecto de la mediación en el aprendizaje intercultural de una lengua del alumnado o a estudiar cómo se puede exponer a los estudiantes de una LE a la variación lingüística que ésta puede presentar en el aula.

\section{Bibliografía}

Butjjes, D.

1991. "Mediating languages and cultures: the social and intercultural dimension restored" en D. Buttjes y M. Byram (eds.) Mediating languages and cultures. Clevedon: Multilingual Matters, 3-16.

Byram, M.

1989. Cultural Studies in Foreign language Education. Clevedon: Multilingual Matters.

1991. "Teaching culture and language: towards an integrated model" en D. Buttjes y M. Byram (eds.) Mediating languages and cultures. Clevedon: Multilingual Matters, 17-33.

Kohler, M.

2015. Teachers as Mediators in the FL Classroom. Ontario: Multilingual Matters. Loveday, L. 


\section{A. I. Capel Moreno}

2019. "De la traducción a la enseñanza de lenguas extranjeras como actividades mediadas culturalmente" Sabir. International Bulletin of Applied Linguistics, 1: 38-51

1982. The Sociolingüistics of Learning and using a Nonnative Language. Oxford: Pergamon.

Nostrand, H. L.

1991. "Basic Intercultural Education Needs Breadth and Depth: The Role of a Second Culture" en E. Silver (ed.) Critical Issues in Foreign Language Instruction. Nueva York: Ed. Garland Publishing, 131-159.

Ortega Arjonilla, E.

2004. "La traducción como actividad mediada culturalmente" en F. J. García Marcos et alt. (eds.) Traducción, Cultura e Inmigración. Reflexiones Interdisciplinares. Granada: Ed. Atrio, 61-74.

Paricio Tato, $\mathrm{M}^{\mathrm{a}} \mathrm{S}$.

2014. "Competencia intercultural en la enseñanza de lenguas extranjeras" en Porta Linguarum. Enero 2014: 215-226.

Quine, W.V.O.

1959. "Significado y traducción" en Luis M.L. Valdés Villanueva (ed.) La búsqueda del significado. Barcelona: Ed. Tecnos, 244-270.

Wierlacher, A. (ed.)

1987. Perspektiven und Verfahren interKultureller germanistik. Munich: Iudicium. Zarate, G.

1986. Enseigner une culture étrangere. Paris: Ed. Hachette. et al.

2004. Cultural mediation in language learning and teaching. Estrasburgo: Consejo de Europa. 